\title{
Endoscopic Endonasal Resection of Adenoid Cystic Carcinoma of the Sinonasal Tract and Skull Base
}

\author{
Luca Volpi, MD, PhD; Maurizio Bignami, MD, FACS; Davide Lepera, MD; \\ Apostolos Karligkiotis, MD; Andrea Pistochini, MD, PhD; Giorgia Ottini, MD; Elena Grigioni, MD; \\ Davide Lombardi, MD; Piero Nicolai, MD; Paolo Castelnuovo, MD, FRCS(Ed), FACS
}

Objectives: Adenoid cystic carcinoma (ACC) is a locally aggressive salivary gland malignancy prone to perineural invasion and local recurrences. In the literature, few data exist to guide treatment when this tumor involves the paranasal sinuses and skull base. We report our experience in the management of sinonasal adenoid cystic carcinoma through an endoscopic endonasal approach.

Methods: Retrospective analysis of patients affected by sinonasal ACC treated through an endoscopic endonasal approach from 1997 to 2015, managed at the Universities of Varese and Brescia, Italy.

Results: Thirty-four patients were included in the analysis. The ethmoid sinus (55.9\%), nasal septum (17.7\%), maxillary sinus $(11.7 \%)$, and sphenoid sinus $(5.9 \%)$ were the primary tumor sites encountered. Twenty patients (58.8\%) presented with T3 or T4, without any systemic spreading. Twenty-nine patients underwent endoscopic transnasal resection, whereas the involvement of the anterior skull base in five cases required a transnasal endoscopic craniectomy. Overall, 20 of 34 (58.8\%) patients received some form of adjuvant radiotherapy. The follow-up ranged from 12 to 202 months (mean of 73.2 months). The 5-year overall, disease-specific, and recurrence-free survival rates were $86.5 \% \pm 7.39 \%, 86.5 \% \pm 7.39 \%$, and $71.8 \% \pm 8.67 \%$, respectively.

Conclusions: The endoscopic approach is safe and effective for selected sinonasal ACC, reducing the comorbidities of the external approaches while producing similar oncological results. High T-stage, grade III histology, positive surgical margins, and perineural infiltration all have an important negative prognostic value.

Key Words: Adenoid cystic carcinoma, endoscopy, sinonasal, skull base.

Level of Evidence: 4.

Laryngoscope, 00:1-7, 2018

\section{INTRODUCTION}

Sinonasal cancers account for approximately $4 \%$ of all head and neck malignancies. ${ }^{1-3}$ Adenoid cystic carcinomas (ACC) account for $5 \%$ of all sinonasal malignancies. ${ }^{4,5}$

ACC is the most common salivary gland tumor and presents different histological growth patterns (solid, tubular, and cribriform), which are related with prognosis. ${ }^{6}$ The biological behavior of ACCs is unique. These tumors have a propensity for perineural spreading through the skull base, cavernous sinus, and orbit. Longterm survival is poor, with most patients dying of local

From the Department of Biotechnology and Life Sciences (L.V., M.B., D.L, A.K., A.P. P.C.); Head and Neck Surgery and Forensic Dissection Research Center (L.V., M.B., D.L, A.K., A.P. P.C.); Department of Biotechnology and Life Sciences, Department of Surgical and Morphological Sciences (G.o.); Unit of Pathology, and Department of Oncology (E.G), University of Insubria-Varese, ASST Sette Laghi, Ospedale Circolo Macchi, Varese, Italy; Department of Otorhinolaryngology (D.L, P.N.), University of Brescia, Brescia, Italy

Editor's Note: This Manuscript was accepted for publication on July 11, 2018.

Financial Disclosure: The authors have no funding, financial relationships, or conflicts of interest to disclose. Informed consent was obtained from all the subjects involved in this survey.

Send correspondence to Davide Lepera, MD, Department of Otorhinolaryngology, University of Insubria, Varese, Via Guicciardini 9, Varese, Italy. E-mail: davide.lepera86@gmail.com

DOI: 10.1002/lary.27485 recurrence rather than from distant metastases. ${ }^{7,8}$ These features make the surgical management particularly challenging, and the treatment of ACCs is currently a matter of debate.

The endoscopic endonasal technique permits a safe and feasible removal in selected cases, with promising oncologic outcomes and lower morbidity than with the external approaches. ${ }^{9}$ Moreover, adjuvant treatments can be associated to improve survival and reduce the risk of recurrences. $^{10}$

The aim of this study was to analyze the clinical features, surgical treatment, and the survival outcomes in patients affected by ACC and treated endoscopically.

\section{MATERIALS AND METHODS}

\section{Patient Population}

A retrospective review of patients affected by sinonasal ACC and treated at the otorhinolaryngology department of the Universities of Insubria and Brescia between June 1997 and December 2015 was performed. Institutional review board approval was obtained.

The patients included were affected by sinonasal and nasopharyngeal ACCs treated with radical intent through an endoscopic approach. Exclusion criteria were the external approaches, unresectable disease (involvement of cavernous sinus, internal 
carotid artery, optic nerve, orbital apex, brain, clivus), and endoscopic debulking for relief of symptoms.

Epidemiological and clinical data, surgical reports, radiology, complications, adjuvant therapies, and follow-up information were reviewed. All patients received a preoperative computed tomography (CT) and magnetic resonance imaging with gadolinium to evaluate the tumor extension (brain invasion, perineural spread, and to differentiate between inflammatory and neoplastic tissues). Multiple biopsies under endoscopic control were performed. All patients completed their preoperative staging with a total-body CT scan with enhancement and a neck ultrasound. The patients included were retrospectively staged according to the 2010 UICC TNM staging system (7th Edition) ${ }^{11}$

\section{Surgery}

All patients were informed about the possibility of switching intraoperatively to a combined cranioendoscopic resection. The surgical procedures were tailored to the extension of the tumor together with the patients' general health and comorbidity. The resection was limited to the sinonasal complex (endoscopic endonasal resection) for initial stage tumors. In the case of involvement of the anterior skull base, the resection was extended to include the ethmoidal roof and the dura mater of the anterior skull base from the posterior wall of the frontal sinus to the planum sphenoidalis and between the medial orbital walls (endoscopic resection with transnasal craniectomy [ERTC]). In such cases, transnasal skull base reconstruction was performed according to a multilayer technique, using autologous materials (fascia lata or iliotibial tract). Endoscopic nasopharyngectomy was indicated in the case of infiltration of the nasopharynx. ${ }^{12}$ When the tumor was extended to the pterygopalatine fossa (PPF) or the medial wall of the maxillary sinus, the surgery included the resection of the PPF content or an endoscopic maxillectomy, respectively. In all cases, the surgical margins were examined intraoperatively with frozen sections, and the resection was enlarged until clear margins were obtained or if further resections were impossible to achieve regardless of the approach. The indications, contraindications, and specific surgical techniques of the different approaches have been detailed previously. ${ }^{13-15}$

Postoperative treatments included adjuvant radiotherapy (RT) in cases of close or positive surgical margins; perineural invasion or involvement of critical regions such as frontal sinus, sphenoid sinus, lamina papyracea or periorbit; pterygopalatine fossa; and anterior skull base (with or without dural infiltration). The postoperative RT was delivered using the three-dimensional conformal RT or intensity modulated RT (IMRT) or particle $\mathrm{RT}$. The clinical target volume usually consisted in the whole resection cavity and involved subsites and the possible pathways of spreading.

\section{Follow-up}

Life-long follow-up is necessary. In our cohort, postoperative follow-up was performed with nasal endoscopy every 15 days until the complete healing of the surgical cavity; then, every month during the first year, every 4 months during the second year, and every 6 months thereafter. Postoperative magnetic resonance (MR) with gadolinium was performed after 4 months after surgery and thereafter every 6 months. A systemic staging with total-body CT scan was performed once a year. No patients were lost during the follow-up.

\section{Histopathology}

Pathological slides were revised independently by two experienced pathologists and were classified according to Perzin grading system. ${ }^{6}$ It foresaw three grades: I was a predominantly tubular pattern without solid component, II was a predominantly cribriform pattern with a maximum $30 \%$ of solid pattern, and III was more than $30 \%$ of solid pattern.

The revision of the surgical resection specimens also evaluated the perineural, bony, dural, orbital, and brain invasion to confirm the stage (UICC 2010 TNM staging system 7th Edition). ${ }^{11}$

\section{Statistical Analysis}

All data were collected and processed with a commercially available computer software package (SPSS for Windows, version 19; IBM Corp., Armonk, NY). The estimated distribution of the overall survival (OS), disease-specific survival (DSS), and RFS were calculated using the Kaplan-Meier method $(P$ values $<0.05$ were considered significant).

\section{RESULTS}

\section{Patients}

A total of 34 patients fulfilled the inclusion criteria. The clinical and epidemiological data are summarized in Table I. No gender prevalence was found, and the average age was 56.8 years old (18-94).

The most reported symptoms were unilateral nasal obstruction (66\%), epistaxis (40\%), and headache $(30 \%)$. Only two patients were asymptomatic.

Eight patients (24\%) referred to our departments after previous treatments: one patient had RT; three patients had surgery plus RT; and four patients had surgery.

The tumors originated from the nasal cavity and ethmoid in 28 cases $(6 \mathrm{~T} 1,3 \mathrm{~T} 2,6 \mathrm{~T} 3,4 \mathrm{~T} 4 \mathrm{a}$, and $9 \mathrm{~T} 4 \mathrm{~b})$, from the maxillary sinus in four cases ( $3 \mathrm{~T} 1$ and $1 \mathrm{~T} 2$ for the extension to the ethmoid), and from the nasopharynx in two cases staged as T2 and T4. Only a T2 tumor of the maxillary sinus was $\mathrm{N} 1$ at the time of the initial diagnosis.

The Perzin grade II was the most reported (23 cases, $67.6 \%$ ), followed by grade III (7 cases, $20.6 \%$ ) and grade I (4 cases, $11.8 \%$ ).

\section{Treatment, Perioperative Outcomes, and Complications}

Twenty-nine patients underwent ER, and in five cases an ERTC was performed due to the involvement of the anterior skull base. When the maxillary sinus was involved (only T1 and T2 were included), we performed an endoscopic medial maxillectomy. The two patients presenting involvement of the nasopharynx underwent endoscopic nasopharyngectomy type II (T2) and type III (T4). There were no major perioperative complications.

Seven patients $(20.6 \%)$ had positive surgical margins on histological examination (R1) of the operative specimen in sites not amenable for further surgical 
TABLE I.

Clinical and Epidemiological Data of the Included Patients

\begin{tabular}{|c|c|c|}
\hline Parameter & & $\begin{array}{c}\text { No. of } \\
\text { Patients (\%) }\end{array}$ \\
\hline Median age, y & $56.8(18-94)$ & 34 \\
\hline \multirow[t]{2}{*}{ Gender } & Male & $15(44.1)$ \\
\hline & Female & $19(55.9)$ \\
\hline \multirow[t]{2}{*}{ Disease presentation } & Initial & $26(76.5)$ \\
\hline & Recurrent & 8 (23.5) \\
\hline \multirow[t]{5}{*}{ Tumor epicenter } & Ethmoid sinus & $19(55.9)$ \\
\hline & Nasal septum & $6(17.7)$ \\
\hline & Maxillary sinus & $4(11.7)$ \\
\hline & Sphenoid sinus & $2(5.9)$ \\
\hline & $\begin{array}{l}\text { Other (nasopharynx, } \\
\text { pterygoid) }\end{array}$ & $3(8.8)$ \\
\hline \multirow{3}{*}{$\begin{array}{l}\text { Histological classification (Perzin } \\
\text { Grading System) }\end{array}$} & Grade I & $4(11.8)$ \\
\hline & Grade II & $23(67.6)$ \\
\hline & Grade III & $7(20.6)$ \\
\hline \multirow[t]{2}{*}{ Treatment } & Surgery & $14(41.2)$ \\
\hline & Surgery + RT & $20(57.8)$ \\
\hline \multirow[t]{4}{*}{ T classification } & $\mathrm{T} 1$ & $9(26.5)$ \\
\hline & T2 & $5(14.6)$ \\
\hline & T3 & $6(17.7)$ \\
\hline & T4 & $14(41.2)$ \\
\hline \multirow[t]{2}{*}{$\mathrm{N}$ classification } & No & $33(97.1 \%)$ \\
\hline & $\mathrm{N}+$ & $1(2.9)$ \\
\hline \multirow[t]{2}{*}{ Distant metastasis } & MO & 34 (100\%) \\
\hline & M1 & 0 \\
\hline \multirow[t]{2}{*}{ Surgical margins } & Positive & 7 (20.6) \\
\hline & Negative & $27(79.4)$ \\
\hline \multirow[t]{2}{*}{ Perineural invasion } & Present & $11(32.3)$ \\
\hline & Absent & $23(67.7)$ \\
\hline Follow-up (months) & Range & 12-202 \\
\hline
\end{tabular}

$\mathrm{N}=$ node; $\mathrm{RT}$ = radiotherapy; $\mathrm{T}=$ tumor.

resection ( 4 cases on the Vidian nerve and 3 on maxillary nerve).

A postoperative RT was performed in 20 patients (58.8\%) due to advanced-stage disease and close or positive surgical margins, whereas 14 patients did not require RT because of early-stage disease and free-margins resection. The only patient with $\mathrm{N} 1$ stage underwent neck dissection.

The mean follow-up was 73.2 months. At the time of this study, 25 patients $(73.5 \%)$ were free from disease (NED); five patients (14.7\%) had died of disease (DOD); and four patients $(11.8 \%)$ were alive with disease (AWD).

Ten patients $(29.4 \%)$ developed recurrence, with a mean time to recurrence of 44.7 months (range 8-128). These patients were staged primary tumor (pT)1 (R0) in one case, pT2 (R0) in two cases, pT3 (R0) in one case, pT4a (R0 and R1) in two cases, and pT4b in four cases (3 cases $\mathrm{R} 0$ and $1 \mathrm{R} 1$ ).

Six recurrences were intracranial. Seven of 10 developed only a local recurrence, three of 10 also developed distant metastases (two lung metasteses and one lung and liver metastases), but no patients had regional metastasis. Two of these 10 patients were NED, four were AWD, and four were DOD.

\section{Oncological Outcomes}

In our cohort, the 5-year OS and the DSS rates were both $86.5 \% \pm 7.39 \%$, whereas the 10-year OS and DSS were both $66.8 \% \pm 1.37 \%$ (Fig. 1A-Fig. 1B). Overall survival and DSS data are equal because there are no patients who had died from other causes, and five patients were DOD. The 5-year OS and DSS depending on surgical margins were both $93.7 \% \pm 6.05 \%$ for the free-margins resection versus $41.7 \% \pm 3.04 \%$ for positive margins $(P=0.0144)$ (Fig. $2 \mathrm{~A}-$ Fig. 2B). In addition, the perineural infiltration was analyzed and we found that it is related with a long-term failure because the 5-year OS and DSS are similar $(87.7 \% \pm 8.2 \%$ without perineural infiltration; $88.9 \% \pm 1.05 \%$ for patients with perineural infiltration). Conversely, the 10-year OS and DSS were lower for the group with perineural infiltration $(75.2 \% \pm 1.36 \%$ vs. $44.4 \% \pm 3.19 \%$, respectively). Nevertheless, the Kaplan-Meier curves were not statistically different $(P=0.144)$ (Fig. 2C-Fig. 2D).

The 5-year OS and DSS depending on treatment were both $88.9 \% \pm 1.05$ for surgery plus RT versus $84.8 \% \pm 0.09$ for surgery alone. On the other hand, the 10 -year OS and DSS were $88.9 \% \pm 1.05 \%$ for surgery plus RT versus $58.2 \% \pm 1.72 \%$ for surgery alone $(P=0.056)$ (Fig. 3D-Fig. 3E) and was close to statistical significance.
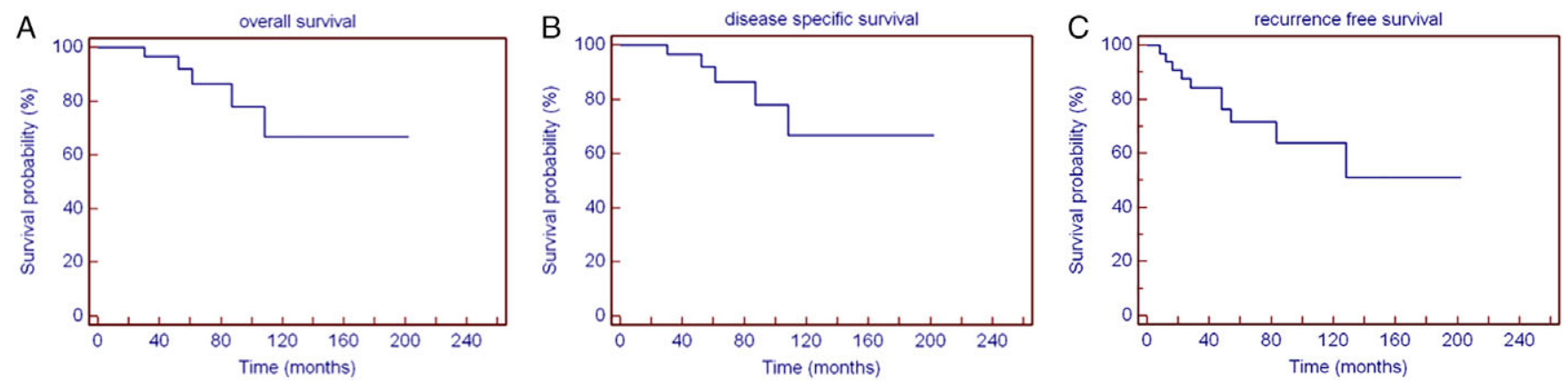

Fig. 1. Overall (A), disease-specific $(B)$, and recurrence-free $(C)$ survival of the included population. [Color figure can be viewed in the online issue, which is available at www.laryngoscope.com.] 

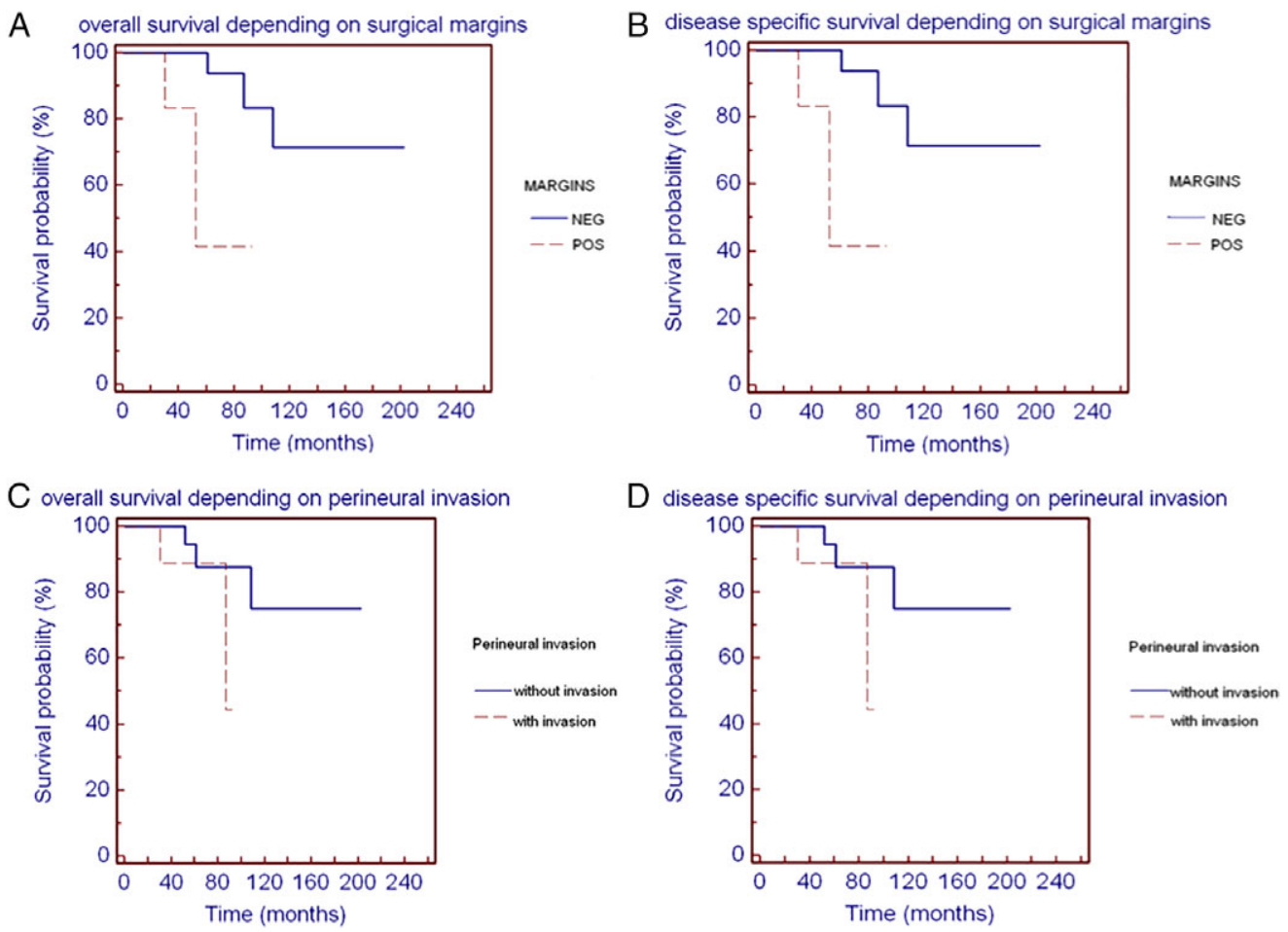

Fig. 2. Overall and disease-specific survival depending on the surgical margins (A-B) and according on the perineural invasion (C-D). [Color figure can be viewed in the online issue, which is available at www.laryngoscope.com.]

Five-year recurrence-free survival (RFS) was $71.8 \% \pm 8.67 \%$ due to the occurrence of a local relapse in 10 patients (Fig. 1C). We analyzed the RFS comparing the T-stage of the tumor and the treatment. It is evident that recurrences are related with the stage, with a 5-year RFS of $83.3 \% \pm 1.52 \%$ for $\mathrm{T} 1,75 \% \pm 2.17 \%$ for $\mathrm{T} 2,50 \% \pm$ $3.54 \%$ for $\mathrm{T} 3,66.7 \% \pm 2.72 \%$ for $\mathrm{T} 4 \mathrm{a}$, and $62.3 \% \pm 1.98 \%$ for T4b $(P=0.83)$ (Fig. 4).
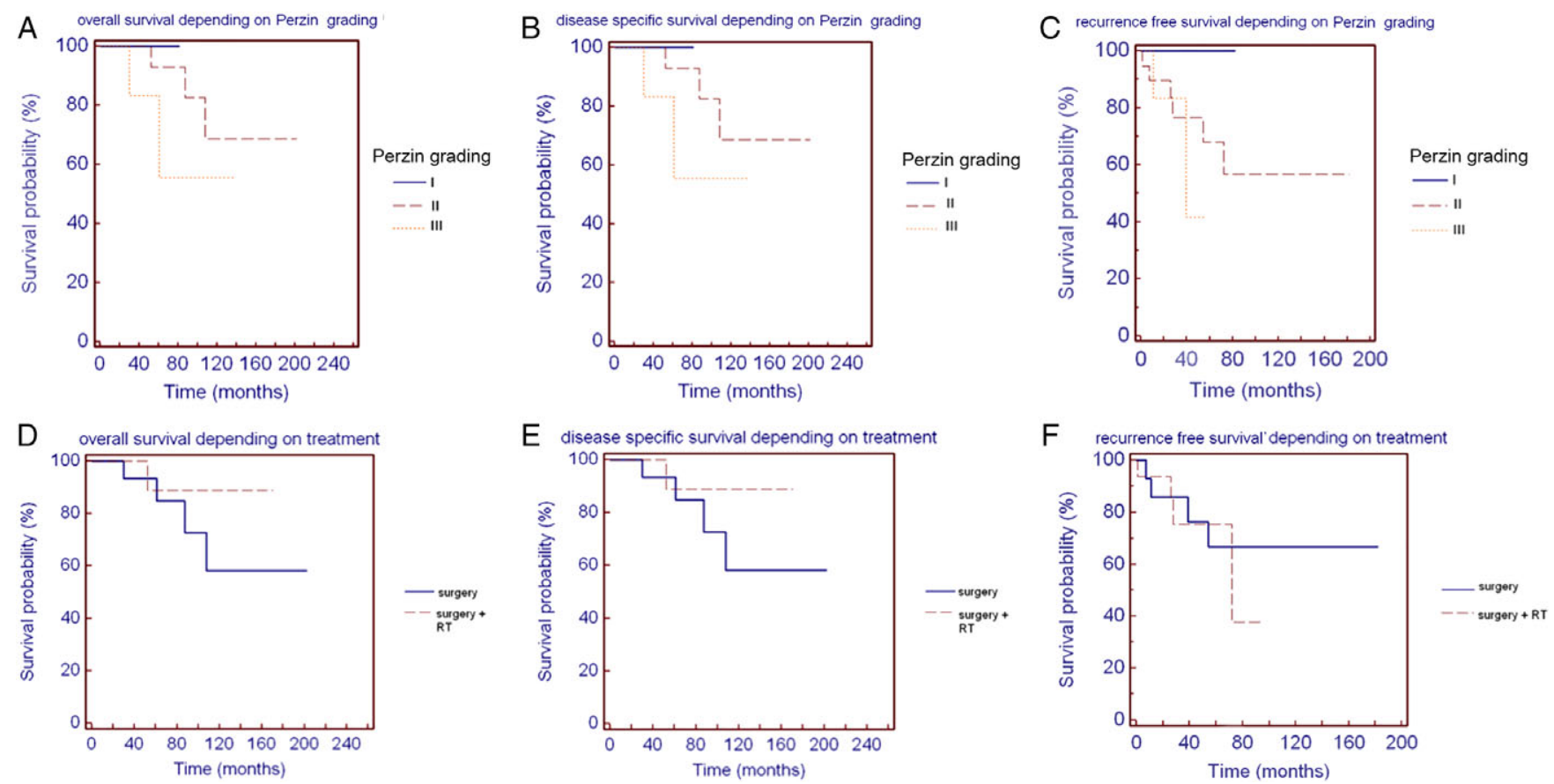

Fig. 3. Overall, disease-specific and recurrence-free survival depending on Perzin grade (A-B-C) and according on the treatment (D-E-F). [Color figure can be viewed in the online issue, which is available at www.laryngoscope.com.] 


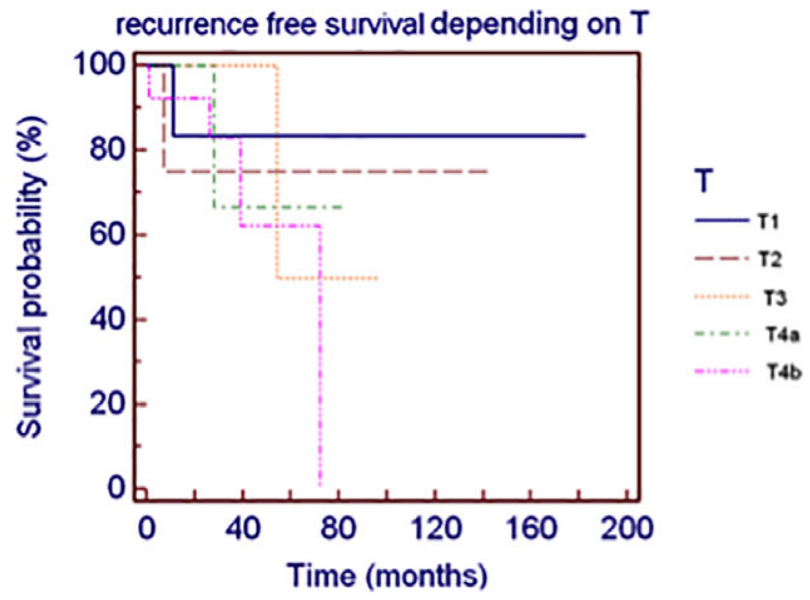

Fig. 4. Recurrence-free survival depending on T stage. [Color figure can be viewed in the online issue, which is available at www.laryn goscope.com.]

Five-year RFS depending on treatment were $66.7 \% \pm 1.39 \%$ for the surgery alone and $75.2 \% \pm 1.29 \%$ for surgery plus RT $(P=0.04)$ (Fig. $3 \mathrm{~F})$.

Analysis of the 5-year OS and DSS according to the Perzin grading system (Fig. 3A-Fig. 3B) resulted in 100\% for grade I, $92.9 \% \pm 0.68 \%$ for grade II, and $55.6 \% \pm 0.24 \%$ for grade III $(P=0.035)$. The 5 -year RFS depending on the Perzin grading system was $100 \%$ for grade I, $68 \% \pm 1.23 \%$ for grade II, and $41.7 \% \pm 3.04 \%$ for grade III $(P=0.041)$ (Fig. 3 C).

\section{DISCUSSION}

An ACC is a slow-growing tumor with a propensity for perineural spreading and intracranial extension. ${ }^{16-18}$ A free-margin surgical resection is difficult to achieve in the sinonasal-nasopharyngeal tract because of the critical relationship with vital structures, the presence of cranial nerves, and the initial asymptomatic course of the disease ${ }^{19}$; therefore, prognosis of ACC of the sinonasal tract is poor. ${ }^{20}$ The clinical behavior of ACC can be considered a paradox: the tumor growth is slow; however, its clinical course is relentless; multiple local recurrences are the rule; and the metastatic spreading to regional lymph nodes is uncommon, although distant spread to the lungs and bones is frequent. Finally, the 5-year survival rates are high, but 10- to 20-year survival rates are dramatically low. ${ }^{21}$

ACC is traditionally classified into three different subtypes: tubular, cribriform, and solid-with the last one being related with the worst prognosis. However, in most of the cases the three different patterns coexist. Perzin et al. ${ }^{6}$ in 1978 proposed a histopathological classification dividing ACCs according to the prevalence of the three different patterns: predominance of tubular component without a solid part is classified as grade I; predominance of cribriform pattern with at least $30 \%$ of solid pattern is classified as grade II; and more than $30 \%$ of solid pattern is classified as grade III. In our analysis, the worst prognoses and RFS were found for grade III tumors when compared with grade I through II. Grade III ACC should be considered as a high-grade tumor due to its more aggressive growth pattern and tendency for early development of distant metastases, ${ }^{22-25}$ and it should be viewed as a specific entity within the group of ACCs.

Regarding the survival rates, the 5-year OS reported in the literature for the patients affected by sinonasal ACC is between $86 \%$ and $50 \% .{ }^{26}$ In our study, 5-year and 10 -year OS were $86.5 \%$ and $66.8 \%$, respectively.

Surgery with or without adjuvant RT is the most common treatment modality, and the ideal treatment paradigm has yet to be defined. ${ }^{27}$ An extensive preoperative radiological study with a $\mathrm{CT}$ scan and MR permits defining the limits of the tumor and deciding between a craniofacial resection and an endoscopic approach. These approaches are not in contrast with each other but present different indications for achieving a free-margin resection. Endoscopic transnasal resection of sinonasal malignancies has been demonstrated to preserve the quality of life with oncological results similar to those achieved with external approaches. ${ }^{28}$ The absence of facial incisions and osteotomies, the visualization of tumor borders, less postoperative pain, shorter hospitalization time, better quality of life, and reduced intraoperative mortality are the major advantages promoting the endoscopic approach as a good alternative to the traditional external procedures. ${ }^{29}$ However, when major vessels need to be crossed or a free-margin resection cannot be achieved, a combined endoscopic-craniofacial approach must be planned.

The goal of surgery is a free-margin resection, even if it is critical due to the submucosal-subperiosteal diffusion and perineural spreading, making intraoperative frozen sections on the resection margins mandatory. In particular, a radical resection is difficult when the tumor involves the pterygopalatine fossa, infratemporal fossa, and superior parapharyngeal space, where it runs along the nerves into the intracranial space. In our analysis, a microscopic negative margin demonstrated a statistically significant improvement of OS, in accordance with the literature. $^{30}$

Postoperative radiation seems to provide the best OS, as reported in recent studies. ${ }^{27,31}$ Miglianico et al. found a local control rate of $77.8 \%$ at 5 years for patients treated by surgery plus RT against $44 \%$ for patients treated by surgery alone. Lupinetti et al. evidenced a significant difference in disease-specific survival $(P=0.05)$. Our 5 -year and 10-year OS rates, depending on treatment (surgery plus RT vs. surgery), were respectively $88.9 \%$ versus $84.8 \%$ ( 5 year) and $88.9 \%$ versus $58.2 \%$ (10 year), confirming the long-term data of the literature. The majority of authors describe a combination of primary surgery, followed by adjuvant RT as the recommended treatment. However, the benefit of adjuvant RT has never been demonstrated clearly. ${ }^{26}$ Postoperative radiation may be delivered using conventional photon RT (e.g., IMRT) or taking advantage of the recently introduced particle therapy, especially carbon ion therapy, which has shown promising rates of local control as adjuvant treatment and also for inoperable cases. ${ }^{32}$ Ramaekers et al. showed that the 5-year local control rate after proton therapy is 
higher than achieved with IMRT for cases of sinonasal cancer ( $88 \%$ vs. $66 \%, P=0.035$ ). The limited data available indicate that toxicity tends to be lower for carbon-ion and proton compared to photon $\mathrm{RT}^{33}$

According to our data, perineural infiltration influences the survival rates: 10-year OS without infiltration was $75.2 \%$, whereas 10 -year OS with infiltration was $44.4 \%$ even if no a clear statistically significant difference was evident $(P=0.144)$. According to Garden et al., the perineural infiltration is in relation not only with the overall survival but also with local recurrence of the disease. In that study, Garden et al. showed how patients either with or without perineural infiltration have failure rates of $18 \%$ and $9 \%$, respectively $(P=0.02) .^{23}$

Local recurrence rather than distant metastases is the main event in ACC's history and develops in a significant percentage of patients $(65 \%) .{ }^{27}$ According to the literature, advanced stages of disease, perineural invasion, positive margins of surgical resection, and omission of postoperative radiation are independent predictors of local recurrence. ${ }^{26}$ Our results indicate that histopathological grade, $\mathrm{T}$ stage, and the treatment all influence local recurrence. Maxillary sinus, sphenoid sinus, and nasopharynx are associated with a significant percentage of relapses, probably due to the close relationship with major nerve (Vidian, V2, V3). Lupinetti et al., in their retrospective review, show how patients with nasal cavity tumors have the best survival, whereas patients with sphenoid tumors have the worst survival. ${ }^{27}$

In contrast to sinonasal squamous cell carcinoma, lymphatic spreading to the neck plays a minor role in the spread of ACC. Nodal involvement in this histology with this location is rare $(0-5 \%){ }^{8,34}$ In our study, only one patient ( 1 of 34 ) showed a regional nodal metastasis at the time of diagnosis, and no patient developed neck metastases during follow-up.

Distant metastase at diagnosis are also rare, with reported rates of $1 \%$ to $3 \% .^{8,34}$ The most frequent sites are lung, bone, liver, and brain. In our study, none of the patients presented distant metastases at the time of diagnosis. However, three patients developed distant metastases during follow-up; one patient had liver and lung metastases after 16 months and is alive with disease 33 months after surgery. Two patients had lung metastases; one is alive with disease after 33 months, and the other was DOD after 61 months.

As reported in literature, the biological behavior of the primitive cancer as reported in literature permits a prolonged survival also in metastatic patients, particularly in cases with lungs metastasis. ${ }^{34,35}$ These findings explain how the presence of metastases should not exclude correct treatment of the primary site. At the time of this study, our data are sufficient for a discussion on this topic.

\section{CONCLUSION}

Our study demonstrates how the prominent issue in sinonasal ACC is long-term disease control, and the reference treatment should be surgical resection with clear margins followed by adjuvant radiotherapy. The endoscopic approach is safe and effective for selected sinonasal ACC, reducing the morbidities of the external approaches while producing similar oncological results.

High T-stage, grade III histology, positive surgical margins, and perineural infiltration play an important role in negative prognosis.

Radiotherapy is crucial in case of positive surgical margins and perineural spreading, probably delaying the recurrences. Longer follow-up and larger series are needed to better evaluate the role of the endoscopic approach and to understand the biological behavior of the ACC.

\section{BIBLIOGRAPHY}

1. Karligkiotis A, Machouchas N, Bozzo C, et al. Head and neck cancer epidemiology in north Sardinia. Acta Medica Mediterranea 2014;30:41-47.

2. Turner Jh, Reh DD. Incidence and survival in patients with sinonasal cancer: a historical analysis of population-based data. Head Neck 2012;34: 877-885.

3. Myers LL, Nussenbaum B, Bradford CR, et al. Paranasal sinus malignancies: an 18-year single institution experience. Laryngoscope 2002;112: 1964-1969.

4. Lund VJ, Stammberger H, Nicolai P, et al. European position paper on endoscopic management of tumours of the nose, paranasal sinuses and skull base. Rhinol Suppl 2010;1:1-143.

5. Kim KH, Sung MW, Chung PS, et al. Adenoid cystic carcinoma of the head and neck. Arch Otolayngol Head Neck Surg 1994;120:721-726

6. Perzin KH, Gullane P, Clairmont AC. Adenoid cystic carcinomas arising in salivary glands: a correlation of histologic features and clinical course. Cancer 1978;42:265-282.

7. Spiro RH, Huvos, AG, Strong EW. Adenoid cystic carcinoma of salivary origin. Am J Surg 1974;128:512-520.

8. Wiseman SM, Popat SR, Rigual NR, Hicks WL Jr, Orner JB, Wein RO, et al. Adenoid cystic carcinoma of the paranasal sinuses or nasal cavity: a 40-year review of 35 cases. Ear Nose Throat $J$ 2002;81:510-514.

9. Rawal RB, Farzal Z, Federspiel JJ, Sreenath SB, et al. Endoscopic resection of sinonasal malignacy: a sytematic review and meta-analysis. Otolaryngol Head Neck Surg 2016;155:376-386.

10. Erovic BM, Schopper C, Pammer J. Multimodal treatment of patients with minor salivary gland cancer in the case of recurrent disease. Head Neck 2010;32:1167-1172.

11. Sobin LH, Gospodarowizc MK, Wittekind C. International Union Against Cancer (UICC). TNM Classification of Malignant Tumours, Chichester, UK: Wiley; 2011.

12. Liu J, Yu H, Sun X, et al. Salvage endoscopic nasopharyngectomy for local recurrent or residual nasopharyngeal carcinoma: a 10-year experience. In J Clin Oncol 2017;22:834-842.

13. Nicolai P, Battaglia P, Bignami M, et al. Endoscopic surgery for malignant tumors of the sinonasal tract and adjacent skull base: a 10-year experience. Am J Rhinol 2008;22:308-316.

14. Villaret AB, Yakirevitch A, Bizzoni A, et al. Endoscopic transnasal craniectomy in the management of selected sinonasal malignancies. Head Neck 2010;32:786-792.

15. Castelnuovo P, Turri-Zanoni M, Battaglia P, et al. Sinonasal malignancies of anterior skull base: histology driven treatment. Otolaryngol Clin North Am 2016;49:183-200.

16. Chilla R, Schroth R, Eysholdt U, et al. Adenoid cystic carcinoma of the head and neck. Controllable and uncontrollable factors in treatment and prognosis. ORL J Otorhinolaryngol Relat Spec 1980;42:346-347.

17. Moran, JJ, Becker SM, Brady, LW, et al. Adenoid cystic carcinoma. Cancer 1961;14:1235-1250.

18. Smith LC, Lane N, Rankow RM. Cylindroma (adenoid cystic carcinoma). Am. J Surg 1965;110:519-526.

19. Ellington CL, Goodman M, Kono SAG, et al. Adenoid cystic carcinoma of head and neck: incidence and survival trends based on 1973-2007 surveillance, epidemiology and end results data. Cancer 2012;118: 4444-4451.

20. Oplatek A, Ozer E, Agrawal et al. Patterns of recurrence and survival of head and neck adenoid cystic carcinoma after definitive resection. Laryngoscope 2010;120:65-70.

21. Khan AJ, DiGiovanna MP, Ross DA, et al. Adenoid cystic carcinoma: a retrospective clinical review. Int $J$ Cancer 2001;96:149-158.

22. Stallmach I, Zenklusen P, Komminoth P, et al. Loss of heterozygosity at chromosome 6q23-25 correlates with clinical and histologic parameters in salivary gland adenoid cystic carcinoma. Virchows Arch 2002;440: 77-84.

23. Garden AS, Weber RS, Morrison WH, et al. The influence of positive mar gins and nerve invasion in adenoid cystic carcinoma of head and neck treated with surgery and radiation. Int J Radiat Oncol Biol Phys 1995;32: 619-626. 
24. Westra WH. The surgical pathology of salivary gland neoplasms. Otolayngol Clin North Am 1999;39:919-943.

25. Eibling DE, Johnson JT, McCoy JP Jr, et al. Flow cytometric evaluation of adenoid cystic carcinoma: correlation with histologic subtype and survival. Am J Surg 1991;162:367-372.

26. Michel J, Fakhry N, Santini L. Sinonasal adenoid cystic carcinomas: clinical outcomes and predictive factors. Int Oral Maxillofac Surg 2013;42: $153-157$.

27. Lupinetti AD, Roberts DB, Williams MD, et al. Sinonasal adenoid cystic carcinoma - the M.D. Anderson Cancer Center experience. Cancer 2007;110: 2726-2731.

28. Harvey RJ, Winder M, Parmar P, et al. Endoscopic skull base surgery for sinonasal malignancy. Otolaryngol Clin North Am 2011;44: 1081-1140

29. Castelnuovo P, Lepera D, Turri-Zanoni M, et al. Quality of life following endoscopic endonasal resection of anterior skull basew cancers. $J$ Neurosurg 2013;119:1401-1409.
30. Ramakrishna R, Raza SM, Kupferman M, et al. Adenoid cystic carcinoma of the skull base: results with an aggressive multidisciplinary approach. $J$ Neurosur 2016;124:115-121.

31. Miglianico L, Eschwege F, Marandas P, et al. Cervicofacial adenoid cystic carcinoma - study of 102 cases - influence of radiation-therapy. Int $J$ Radiat Oncol Biol Phys 1987;13:673-678.

32. Takagi M, Demizu Y, Hashimoto N, et al. Treatment outcomes of particle radiotherapy using protons or carbon ions as single modality therapy of adenoid cystic carcinoma of the head and neck. Radiother Oncol 2014:113:364-370.

33. Ramaekers BL, Pijls-Johannesma M, Joore MA, et al. Systematic review and meta-analysis of radiotherapy in various head and neck cancers: comparing photons, carbon-ions and protons. Cancer Treat Rev 2011;37:185-201.

34. Rhee CS, Won TB, Lee CH, et al. Adenoid cystic carcinoma of the sinonasal tract: treatment results. Laryngoscope 2006;116:982-986.

35. Sung MW, Kim KH, Kim JW, et al Clinicopathologic predictors and impact of distant metastasis from adenoid cystic carcinoma of the head and neck. Arch Otolaryngol Head Neck Surg 2003;129:1193-1197. 